\title{
Treatment of Myelodysplastic Syndrome Transforming into Acute Myelogenous Leukemia with Azacytidine: A Retrospective Study from Cancer Centre in Northern India
}

\author{
P. Suresh · A. Sharma $\cdot$ D. Bhurani
}

Received: 13 August 2009/Accepted: 26 July 2010/Published online: 19 October 2010

(C) Indian Society of Haematology \& Transfusion Medicine 2010

\section{Introduction}

Patients more than 55 years of age with Acute Myelogenous Leukemia (AML) are less likely to achieve complete remission and more likely to experience toxicity with conventional induction chemotherapy than younger patients. Azacytidine is the first drug in a new class of compounds classified as DNA hypomethylating agents, to receive FDA approval for the treatment of myelodysplastic syndromes (MDS). At higher doses azacytidine has activity in AML as well.

\begin{abstract}
Aim
To assess the response to azacytidine in patients with MDS transforming to AML who are unable to tolerate standard chemotherapy. Secondary aim was to assess hematological improvement with transfusion independence and the toxicity profile of azacytidine.
\end{abstract}

\section{Materials and Methods}

Five patients were retrospectively identified of MDS transforming to AML who had been treated with azacytidine. All patients had multiple co-morbidities and hence were high risk for chemotherapy complications. Azacytidine

P. Suresh $(\bowtie) \cdot$ A. Sharma $\cdot$ D. Bhurani

Rajiv Gandhi Cancer Institute, Sector 5, Rohini,

Delhi 110085, India

e-mail: psuresh_n@yahoo.com
$75 \mathrm{mg} / \mathrm{m}^{2} /$ day was administered subcutaneously for 7 days every 4 weeks which was defined as one cycle and therapy was continued for as long as response was maintained. Response was assessed by NCI definition of response in AML or by International Working Group (IWG) criteria for hematological improvement in MDS.

\section{Results}

A total of 5 patients were included in this study with a median age of 60 years. 3 were male and 2 female. All of them were MDS transforming into AML. The median duration of therapy with azacytidine was 6 months and the median time to response 3 months (2-5 months). Complete Response (CR) was achieved in one patient (20\%) and Partial Response (PR) in 3 patients $(60 \%)$ and one patient had progressive disease after 3 cycles. This led to an Overall Response Rate (ORR) of $80 \%$ (CR + PR). The median duration of response $(n=4)$ was 6 months (range 3-14). Hematological improvement was noted in all responders. Toxicity was largely manageable in outpatient setting and ECOG performance status in responders remained 1 or less throughout therapy.

\section{Conclusion}

Azacytidine appears to be active in treatment of older patients with MDS transforming to AML who are poor candidates for standard induction chemotherapy. This therapy appears tolerable in poor risk patients and can be managed largely in an out patient setting. Further prospective studies are required to confirm the efficacy and survival advantage with this agent. 